\title{
Dynamic Simulation Including Multipolar Interactions of a System of Dielectric Particles under Electric Field
}

\author{
Annop Limsimarat Non-member (Chulalongkorn University, annop.li@student.chula.ac.th) \\ Boonchai Techaumnat Member (Chulalongkorn University, boonchai.t@chula.ac.th)
}

Keywords : dynamic simulation, dielectrophoretic force, multipole, dielectric particle, ER fluid

Dynamic simulation of particle aggregation under an electric field is performed for an ER fluid, a suspension of dielectric particles in a nonconducting fluid. In the presence of a sufficiently high electric field, the viscosity of ER fluid increases by the change of particle structure into chains and columns aligned in the field direction. Conventionally, the simulations of particles often use a dipole model for computing the interparticle force induced by the electric field. For the simulation in this paper, a multipole model is utilized to improve the calculation of the electric field and the interparticle force. The simulation results by the multipole model are compared to those by the dipole model.

A system of 20 particles, having very low volume fraction $\phi_{V}=0.03$, is simulated to determine the aggregation time that the particles span the electrodes. Figure 1 shows the initial particle positions at $t=0 \mathrm{~ms}$, and the particle positions at $t=300 \mathrm{~ms}$ and $t=240 \mathrm{~ms}$ simulated by the dipole and the multipole models, respectively.

As can be seen from Fig.1, the particles by the multipole model span the electrodes at $240 \mathrm{~ms}$, whereas the particles by the dipole model do not bridge the electrodes at $300 \mathrm{~ms}$. Thus, by using the multipole model, we found that the aggregation time that the particles span the electrodes is significantly shorter.

To investigate characteristics of ER fluids having higher volume fraction $\phi_{V}=0.1$ (67 particles), three systems of different initial positions of particles have been simulated. Figure 2 compares the aggregation of the three systems at $300 \mathrm{~ms}$ when the dipole and the multipole models are used. The results show that the aggregation depends greatly on the initial positions of the particles. However, more consistent structures of aggregation were obtained by the multipole model. Fig. 2 also shows that the alignment of the particles by the multipole model is not well parallel to the field direction as the prediction by the dipole model.

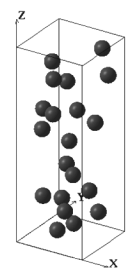

(a)

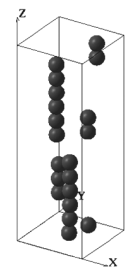

(b)

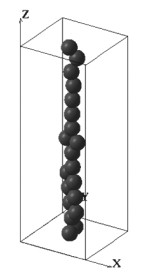

(c)
Fig. 1. System of 20 particles at (a) $0 \mathrm{~ms}$, (b) $300 \mathrm{~ms}$ with the dipole model and (c) $240 \mathrm{~ms}$ with the multipole model

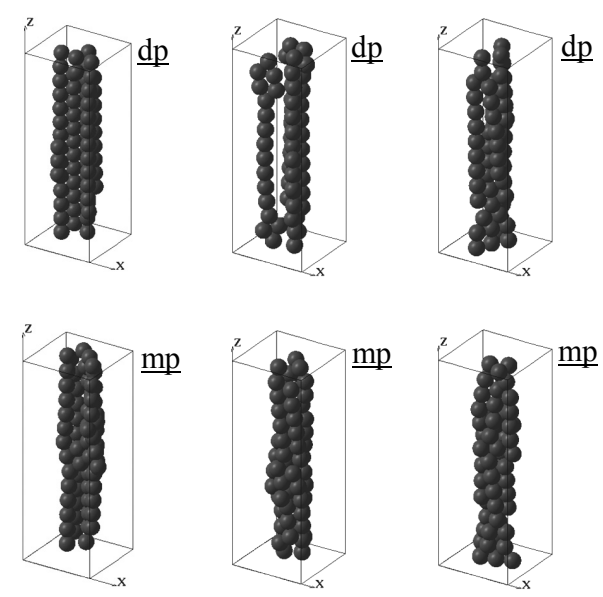

Fig. 2. Three systems (a), (b), and (c) of 67 particles (of different initial states) at $t=300 \mathrm{~ms}$ simulated by the dipole (dp) and the multipole (mp) models

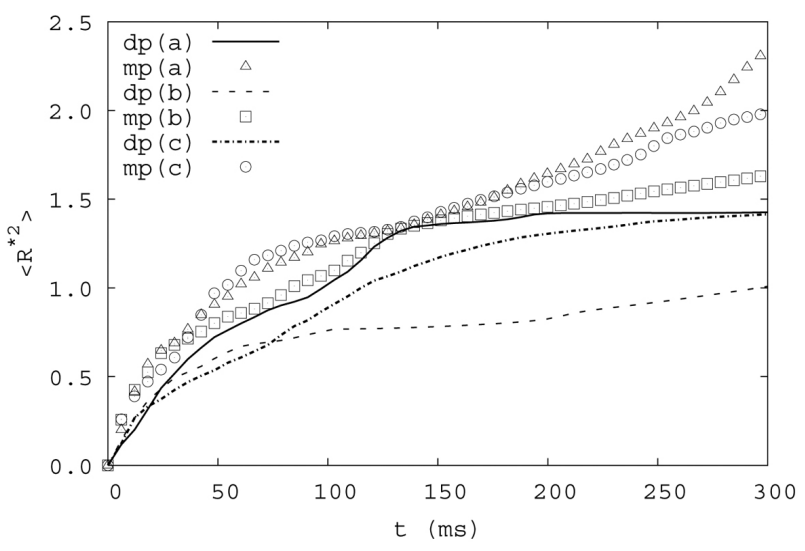

Fig. 3. MSD of the systems of 67 particles

In addition to visual inspection, we have monitored the mean square displacement (MSD), $\left\langle R^{* 2}\right\rangle$, to identify the particle aggregation. In Fig. 3, the MSD is plotted against time. The figure shows that the particles move by a greater distance when the multipole model is used instead of the dipole model. Comparing the values of MSD with the appearance of the particles, we have found that MSD may not be an appropriate index for indicating particle aggregation when the multipole model is used in simulation. 


\title{
Dynamic Simulation Including Multipolar Interactions of a System of Dielectric Particles under Electric Field
}

\author{
Annop Limsimarat* Non-member \\ Boonchai Techaumnat* Member
}

This article presents the dynamic simulation of dielectric particles under an electric field. A multipole model including multipolar interactions between particles is used here to calculate an electric field on the particles and then the force from the electric field. The simulation has been performed on the systems of an ER fluid having different volume fractions. The simulation results by the multipole model have been compared to those by the dipole model, which is often used in the existing works. The comparison shows that, for the system of very low volume fraction, the particles bridge the electrodes faster by the multipole model than by the dipole model. For the systems of higher volume fraction, the aggregation of the particles depends greatly on their initial positions. The results also show that, in a real ER fluid, the ordering of the particle chains may not be parallel well to the field direction as that predicted by the dipole model.

Keywords : dynamic simulation, dielectrophoretic force, multipole, dielectric particle, ER fluid

\section{Introduction}

Study of the particle dynamics under an electric field is important in various applications of dielectric materials. In GIS, for example, the movement of particles may cause a partial discharge, which directly affects the system performance ${ }^{(1)}$. Another example is the ER fluid, which is a suspension of dielectric particles in a nonconducting fluid ${ }^{(2)}$. In the presence of a sufficiently high electric field, the viscosity of ER fluid increases by the change of the particle structure into chains and columns aligned in the field direction. This electromechanical property of the fluid can be used in various applications such as clutches, valves, and other kinds of electrical control devices.

Previous studies on the dynamics of ER fluids often use a dipole model for computing the interparticle force induced by the electric field ${ }^{(3)-(5)}$. However, the force by the dipole model is considerably smaller than the actual force when particles are close to each other. A more accurate model that includes the higher-order terms and utilizes an empirical force function has been proposed ${ }^{(6)}$. There is also a model considering full multipolar interaction for a system of multiparticles ${ }^{(7)}$, but it requires setting up and solving a linear equation system, therefore, not suitable for a system of many particles.

This paper presents dynamic simulation of particle aggregation under an electric field. A multipole model is used in the simulation for calculating the electric field and force on the particles. The positions of the particles varying with time are computed by the Runge-Kutta algorithm. We want to study the particle behavior in an ER fluid and compare the results with those obtained by the conventionally-used dipole model. The simulation presented here should be useful for detailed analysis of the response time of ER fluids, which are often proposed for applications requiring fast response.

\footnotetext{
* Department of Electrical Engineering, Faculty of Engineering, Chulalongkorn University

Phyathi Road, Patumwan, Bangkok 10330 Thailand
}

\section{Physical Configuration}

The systems of particles in this study are represented by a monodisperse suspension of dielectric particles in a host fluid confined between two $L_{x} \times L_{y}$ parallel plate electrodes at $z=0$ and $z=L_{z}$. The particles are treated as spheres having a dielectric constant $\varepsilon_{p}=8$, mass density $\rho=1 \mathrm{~g} / \mathrm{cm}^{3}$, and diameter $\sigma=10 \mu \mathrm{m}$. The dielectric constant of the fluid is $\varepsilon_{f}=$ 2. A uniform external field $E_{0}=3 \mathrm{kV} / \mathrm{mm}$ is applied to the fluid via the parallel electrodes. The viscosity $\eta$ of the host fluid is 0.02 Pa-s. These simulation parameters are referred to an ER fluid of alumina particles in a petroleum oil ${ }^{(4)-(6)}$. The electrode dimensions are $L_{x}=L_{y}=5 \sigma, L_{z}=14 \sigma$, and the number of particles between the electrodes are 20 and 67, respectively, for two cases of different volume fraction.

\section{Methods}

\subsection{Calculation of Dielectrophoretic (DEP) Force}

Force in our systems is the DEP force exerted by a nonuniform field on a polarized but uncharged particle. The force causes the movement and interactions, e.g. repulsion and attraction, between the particles. For approximating the DEP force, we use the following two approaches: (1) the dipole model and (2) the multipole model.

3.1.1 Point-dipole force Consider the interaction force between particle $a$ at $\bar{r}_{a}$ and particle $b$ at $\bar{r}_{b}$, as shown in Fig. 1. The dipole model gives the simplest approximation of the DEP force (3) $^{(3)}$

$$
\bar{F}_{a b}^{d e p}=\frac{3|\bar{p}|^{2}}{4 \pi \varepsilon_{0} \varepsilon_{f}\left|\bar{r}_{a b}\right|^{4}}\left[\left(1-3 \cos ^{2} \theta_{a b}\right) \bar{a}_{r}-\sin \left(2 \theta_{a b}\right) \bar{a}_{\theta}\right],
$$

where $\bar{p}$ is the induced dipole moment, $\bar{r}_{a b}=\bar{r}_{a}-\bar{r}_{b}, \theta_{a b}$ the angle between $\bar{r}_{a b}$ and the z-axis, $\bar{a}_{r}=\bar{r}_{a b} /\left|\bar{r}_{a b}\right|$, $\bar{a}_{\theta}=\bar{a}_{r} \times\left(\bar{a}_{r} \times \bar{a}_{z}\right) /\left|\bar{a}_{r} \times\left(\bar{a}_{r} \times \bar{a}_{z}\right)\right|$, and $\bar{a}_{z}$ is the unit vector in the z-direction, as shown in Fig. 1.

3.1.2 Multipolar force When the particles are close to 


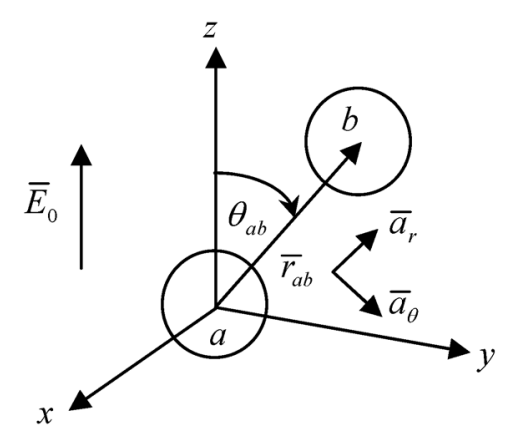

Fig. 1. Coordinate system of the spherical particle pair

each other, the dipolar force is significantly less than the actual force because the higher-order multipolar terms are neglected. The multipole model is a better way to approximate the DEP force. The force, including the multipolar effects, on particle $a$ can be determined by

$$
\bar{F}_{a}^{d e p}=\int_{r=\sigma / 2} \varepsilon_{f} q_{\text {total }}\left[-\nabla\left(\psi_{a}\right)_{e x t}\right] d S_{a}
$$

where $q_{\text {total }}$ is the total effective charge density ${ }^{(8)}$ appearing on the surface of particle $a, S_{a}$ the surface of particle, and $\left(\psi_{a}\right)_{e x t}$ is the electrostatic potential on particle $a$ due to all sources in the exterior of particle $a$. Note that Eq. (2) is a general expression for any dielectric particles. For the two particles in Fig. $1,\left(\psi_{a}\right)_{e x t}$ is the potential on particle $a$ due to the externally applied field and the disturbance produced by particle $b$.

To rewrite Eq. (2) in terms of multipoles, we take the center of particle $a$ as the origin, and express the potentials $\left(\psi_{a}\right)_{I}$ inside and $\left(\psi_{a}\right)_{E}$ outside the particle as in Ref. 9.

$$
\begin{aligned}
& \left(\psi_{a}\right)_{I}=\sum_{j=0}^{N_{m p}} \sum_{k=-j}^{j} L_{j, k} r^{j} \bar{P}_{j,|k|}(\cos \theta) e^{i k \varphi}, \ldots \ldots \ldots \ldots \ldots \ldots \ldots \ldots . . . . . \\
& \left(\psi_{a}\right)_{E}=\sum_{j=0}^{N_{m p}} \sum_{k=-j}^{j}\left[M_{j, k} r^{j}+\frac{B_{j, k}}{r^{j+1}}\right] \bar{P}_{j,|k|}(\cos \theta) e^{i k \varphi},
\end{aligned}
$$

where $N_{m p}$ is the highest order of multipoles, $(r, \theta, \varphi)$ the spherical coordinates, $L_{j, k}, M_{j, k}, \quad$ and $B_{j, k}$ the complex-number coefficients to be determined, and $\bar{P}_{j, k \mid}^{j, k}$ is the normalized associated Legendre function. Note that $L_{j, k}$ and $B_{j, k}$ are related to $M_{j, k}$ so as to satisfy the boundary conditions of the potential and field on the particle ${ }^{(9)}$.

From Eqs. (3) and (4), we can write $q_{\text {total }}$ and $\left(\psi_{a}\right)_{\text {ext }}$ for particle $a$ as

$$
\begin{aligned}
& q_{\text {total }}=\sum_{j=0}^{N_{m p}} \sum_{k=-j}^{j}\left[\frac{\varepsilon_{0}(2 j+1)}{(\sigma / 2)^{j+2}}\right] B_{j, k} \bar{P}_{j,|k|}(\cos \theta) e^{i k \varphi}, \\
& \left(\psi_{a}\right)_{e x t}=\sum_{j=0}^{N_{m p}} \sum_{k=-j}^{j} M_{j, k} r^{j} \bar{P}_{j,|k|}(\cos \theta) e^{i k \varphi} .
\end{aligned}
$$

Note that the dependence on $\sigma, \varepsilon_{p}$ and $\varepsilon_{f}$ is included in the $B_{j, k}$ coefficient. Using Eqs. (2), (5) and (6), the Cartesian components of $\bar{F}_{a}^{\text {dep }}$ can be determined from $B_{j, k}$ and $M_{j, k}$ as follows ${ }^{(10)}$.

$$
\begin{aligned}
& \frac{F_{a, x}^{d e p}}{2 \pi \varepsilon_{0} \varepsilon_{f}}=\sum_{j=0}^{N_{m p}} 2 \operatorname{Re}\left[M_{j+1,1}\right]\left(B_{j, 0}\right) \beta(j+1) \\
& -\sum_{j=1}^{N_{m p}} 2 \operatorname{Re}\left[B_{j, 1}\right]\left(M_{j+1,0}\right) \beta(j) \\
& +\sum_{j=1}^{N_{m p}} 2 \operatorname{Re}\left[\left(B_{j, 1}\right)\left(M_{j+1,-2}\right)\right] \beta(j+2) \\
& +\sum_{j=2}^{N_{m p}} \sum_{k=2}^{j} 2 \operatorname{Re}\left[\left(B_{j, k}\right)\left(M_{j+1,-(k+1)}-M_{j+1,-(k-1)}\right)\right] \\
& \times \beta(j+k+1),
\end{aligned}
$$

$$
\begin{aligned}
& \frac{F_{a, y}^{d e p}}{2 \pi \varepsilon_{0} \varepsilon_{f}}=-\sum_{j=0}^{N_{m p}} 2 \operatorname{Im}\left[M_{j+1,1}\right]\left(B_{j, 0}\right) \beta(j+1) \\
& +\sum_{j=1}^{N_{m p}} 2 \operatorname{Im}\left[B_{j, 1}\right]\left(M_{j+1,0}\right) \beta(j) \\
& +\sum_{j=1}^{N_{m p}} 2 \operatorname{Im}\left[\left(B_{j, 1}\right)\left(M_{j+1,-2}\right)\right] \beta(j+2) \\
& +\sum_{j=2}^{N_{m p}} \sum_{k=2}^{j} 2 \operatorname{Im}\left[\left(B_{j, k}\right)\left(M_{j+1,-(k+1)}+M_{j+1,-(k-1)}\right)\right] \\
& \times \beta(j+k+1),
\end{aligned}
$$$$
\frac{F_{a, z}^{d e p}}{4 \pi \varepsilon_{0} \varepsilon_{f}}=-\sum_{j=0}^{N_{m p}}\left(B_{j, 0}\right)\left(M_{j+1,0}\right)(j+1)
$$$$
-\sum_{j=1}^{N_{m p}} \sum_{k=1}^{j} 2 \operatorname{Re}\left[\left(B_{j, k}\right)\left(M_{j+1,-k}\right)\right]
$$$$
\times \sqrt{(j+1)^{2}-k^{2}},
$$

where $\beta(j)=\sqrt{j(j+1)}$, and $\operatorname{Re}[$ ] and $\operatorname{Im}[$ ] designate the real and imaginary parts of a complex number, respectively.

3.1.3 Calculation of the coefficients $B_{j, k}$ and $M_{j, k}$

We calculate the potential coefficients $B_{j, k}$ and $M_{j, k}$ by using the method of images in an iterative manner similar to the schemes reported in Refs.11-12. The dependences of the force approximation and the calculation time on the highest order of multipoles $\left(N_{m p}\right)$ [for Eqs. (3) to (9)] and the number of iterations $\left(N_{\text {iter }}\right)$ in the method of images were studied by the authors, and the optimal $N_{m p}$ and $N_{\text {iter }} \quad(=4$ and 2, respectively) have been chosen by considering the increasing rate of the force and the simulation time.

3.2 Calculation of Particle Motion Let particle $a$ of mass $m$ be at $\bar{r}_{a}$ in the host fluid at time $t$. The motion of this particle is determined by

$$
m \frac{d^{2} \bar{r}_{a}}{d t^{2}}=-3 \pi \sigma \eta \frac{d \bar{r}_{a}}{d t}+\bar{F}_{a}^{d e p}+\bar{F}_{a}^{r e p}+\bar{F}_{a}^{\text {wall }},
$$

where $\bar{F}_{a}^{d e p}$ is the DEP force as described in Section 3.1, and $\bar{F}_{a}^{\text {rep }}$ and $\bar{F}_{a}^{\text {wall }}$ are the short-range repulsive forces ${ }^{(3)-(5)}$. In simulating the hard-sphere and hard-sphere/hard-wall interactions, both artificial repulsive forces are applied on the particles to avoid overlapping with the other particles and with the electrodes as used in Refs. 3-5. $\bar{F}_{a}^{\text {rep }}$ and $\bar{F}_{a}^{\text {wall }}$ are in exponential forms that decay with distance between the particles and distance between the particle and the electrodes, respectively. The first term on the right hand side of Eq. (10) is the hydrodynamic force ${ }^{(3)-(5)}$. For typical ER fluids, we can neglect the acceleration term on the left hand side of Eq. (10) as the viscosity is predominant. Then, Eq. 
(10) becomes

$$
3 \pi \sigma \eta \frac{d \bar{r}_{a}}{d t}=\bar{F}_{a}^{d e p}+\bar{F}_{a}^{r e p}+\bar{F}_{a}^{\text {wall }}
$$

Applying the following scaling factors:

$F_{0}=\frac{1}{16} \varepsilon_{0} \varepsilon_{f} \alpha^{2} \sigma^{2}\left|\bar{E}_{0}\right|^{2}, \quad t_{0}=3 \pi \eta \sigma^{2} / F_{0}, \quad r_{0}=\sigma \quad$, where $\alpha=\left(\varepsilon_{p}-\varepsilon_{f}\right) /\left(\varepsilon_{p}+2 \varepsilon_{f}\right) ; \quad$ we rewrite Eq. (11) in the dimensionless form as

$$
\frac{d \bar{r}_{a}^{*}}{d t^{*}}=\bar{F}_{a}^{d e p^{*}}+\bar{F}_{a}^{\text {rep* }}+\bar{F}_{a}^{\text {wall }} \text {. }
$$

The symbol * in Eq. (12) denotes dimensionless variables. Using Eq. (12), we compute the positions of particles at a time $t^{*}$ by the fourth-order Runge-Kutta algorithm.

\section{Results}

For the systems of particles in Section 2, the time base $t_{0}=75$ $\mathrm{ms}$. The external field is turned on at $t=0$, and the particles begin to move. The evolution of the particles is calculated with $\Delta t^{*}=8 \times 10^{-5}$ for 50,000 time steps.

A system of 20 particles is simulated to determine the aggregation time that the particles span the electrodes when the volume fraction $\left(\phi_{V}\right)$ is very low, i.e. $\phi_{V}=0.03$. Figure 2(a) shows the initial particle positions at $t=0 \mathrm{~ms}$. Figs. 2(b) and 2(c) show the particle positions at $t=300 \mathrm{~ms}$ and $t=240 \mathrm{~ms}$, respectively, when the dipole and the multipole model are used to calculate the DEP force. The particles are randomly distributed at the initial state in Fig. 2(a). At $t=300 \mathrm{~ms}$, the particles by the dipole model form chains and pairs, but do not bridge the electrodes in Fig. 2(b). On the other hand, the particles by the multipole model at $240 \mathrm{~ms}$ span the electrodes, as shown in Fig. 2(c). Thus, by using the multipole model, the aggregation time that the particles span the electrodes is shorter.

To investigate characteristics of ER fluids having higher volume fraction $\left(\phi_{V}=0.1\right)$, we increase the number of particles to 67 . Three systems of different initial positions of particles have been simulated. An example of the initial state of the particles is shown in Fig. 3.

Figure 4 compares the aggregation of 67 particles of the three systems at $300 \mathrm{~ms}$ when the dipole and the multipole models are used in the simulation. For all cases, the aggregation depends considerably on the initial positions of the particles, as can be seen

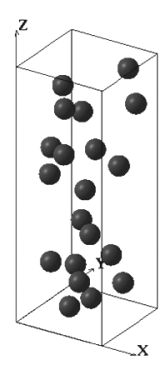

(a)

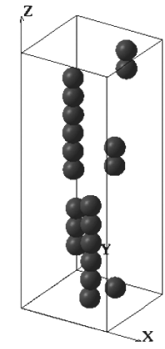

(b)

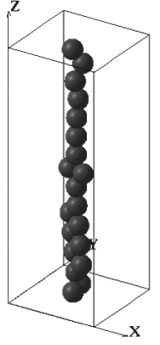

(c)
Fig. 2. System of 20 particles at (a) $0 \mathrm{~ms}$, (b) $300 \mathrm{~ms}$ with the dipole model and (c) $240 \mathrm{~ms}$ with the multipole model from Figs. 4(a) to (c). On the left of Fig. 4(a), the particles by the dipole model arrange themselves very well in the field direction, and the gaps between the particle chains are very small. However, also by the dipole model, the aggregations of the particles on the left of Figs. 4(b) and (c) are not as good as that in Fig. 4(a) because large gaps still exist between the particle chains.

When the DEP force for the simulation is calculated by the multipole model, the alignment of particles in the field (vertical) direction is not as good as that predicted by the dipole model.

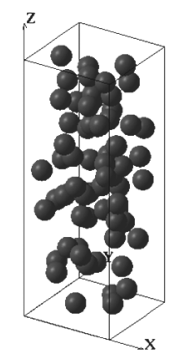

Fig. 3. Randomly distributed particles in a system of 67 particles
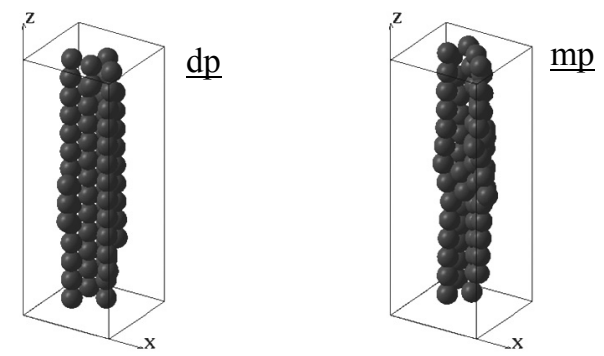

(a)
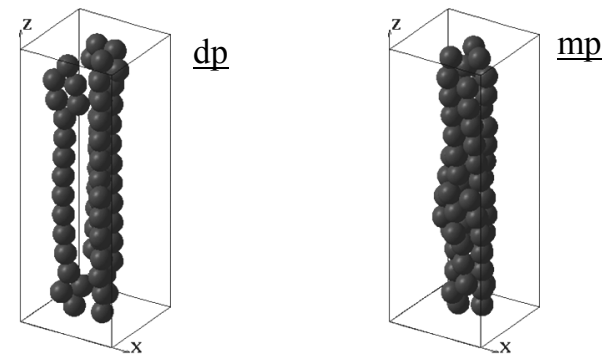

(b)
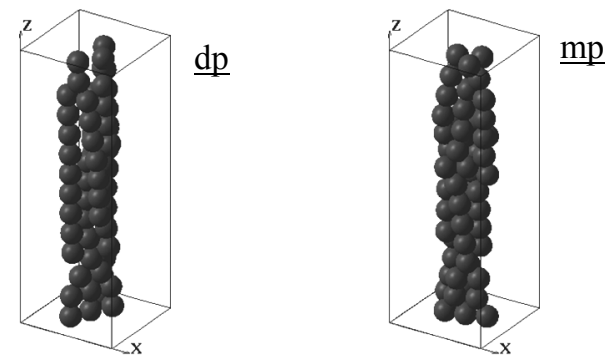

(c)

Fig. 4. Three systems of 67 particles (of different initial states) at $t=300 \mathrm{~ms}$ simulated by the dipole (dp) and the multipole (mp) models 


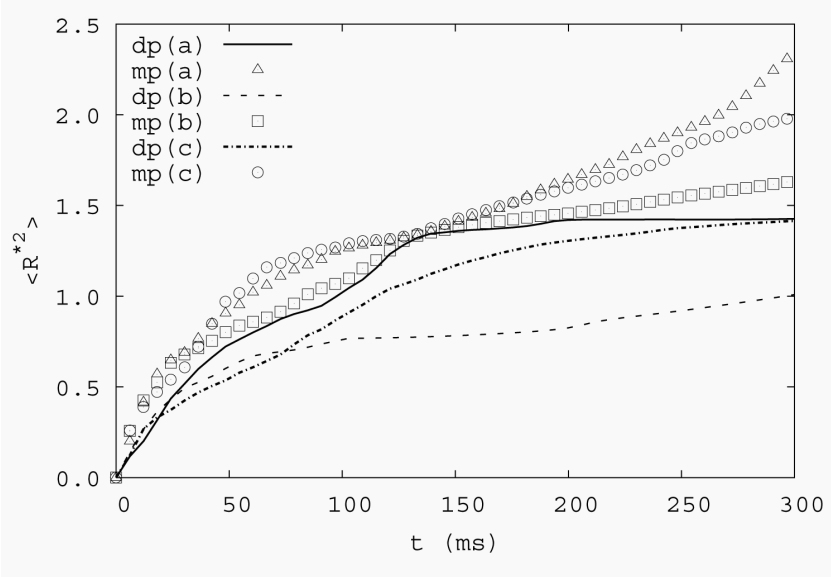

Fig. 5. $\left\langle R^{* 2}\right\rangle$ of the systems of 67 particles

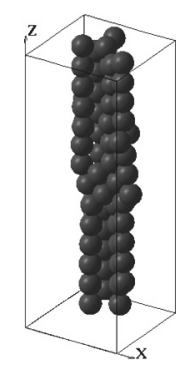

Fig. 6. System (a) at $380 \mathrm{~ms}$ simulated by the multipole model

However, the particles in each system are very close to each other. The gaps between the adjacent particles by the multipole model on the right of Figs. 4(a) to (c) are not clearly seen. Thus, we may conclude that using the multipole model yields more consistent results.

In addition to the visual inspection from Figs. 2 to 4 , we monitor the mean square displacement (MSD) to identify the particle aggregation ${ }^{(3)}$. The $\operatorname{MSD},\left\langle R^{* 2}\right\rangle$, at time $t$ is determined as

$$
\begin{aligned}
& \left\langle R^{* 2}\right\rangle=\frac{1}{N} \sum_{a=1}^{N}\left[R_{a}^{*}(t)\right]^{2}, \\
& {\left[R_{a}^{*}(t)\right]^{2}=\left[\Delta x_{a}^{*}(t)\right]^{2}+\left[\Delta y_{a}^{*}(t)\right]^{2}+\left[\Delta z_{a}^{*}(t)\right]^{2},}
\end{aligned}
$$

where $N$ is the number of particles, $\Delta \xi_{a}^{*}(t)=\xi_{a}^{*}(t)-\xi_{a}^{*}(0)$, and $\xi_{a}^{*}(t)$ is the position of particle $a$ at time $t$ for $\xi=x, y, z$. The MSD is plotted against time in Fig. 5, in which (a), (b) and (c) denote the three different initial positions of particles in reference to Fig. 4.

In Fig. 5, at the beginning state, the MSD of all systems by both models changes rapidly because the particles considerably move to form chains or pairs. By the dipole model, the MSD at $300 \mathrm{~ms}$ approaches the saturation in system (a), but not in systems (b) and (c). This evolution of MSD of system (a) is consistent with the particles in Fig. 4(a) that have a good alignment with the field direction and only small gaps to move. At $300 \mathrm{~ms}$, the slope of MSD by the dipole model for systems (b) and (c) is substantially smaller than that at the initial state. With the multipole model, the
MSD in each system is larger than that by the dipole model, indicating greater amount of particle movement. For all the systems, MSD by the multipole model does not approach saturation, and still clearly increases at $300 \mathrm{~ms}$. However, we have observed that the spatial evolution of particles after $300 \mathrm{~ms}$ is quite slow. For example, Fig. 6 shows the particles in system (a) at $380 \mathrm{~ms}$ by the multipole model. The aggregation differs only slightly from that on the right of Fig. 4(a) at 300 ms. Thus, MSD may not be appropriate as an index for indicating aggregation when the multipole model is used in simulation.

\section{Conclusions}

We have performed the simulation on the systems of particles with parameters referred to a real ER fluid. The multipole model is used here to improve the approximation of the force induced on the particles due to the electric field. When comparing the results to those by the conventional dipole model, we can conclude as follows: (1) For the system of 20 particles, the time by the multipole model for the particles to bridge the electrodes is significantly shorter. (2) For the systems of 67 particles, where the average separation between the particles is smaller, we have found that (2.a) the aggregation of the particles depends greatly on their initial positions; however, the resultant aggregation becomes more consistent, (2.b) the particles by the multipole model move by a greater distance, and (2.c) the ordering of particles in the field direction is not as good as that obtained by the dipole model.

\section{Acknowledgment}

This work is supported by the Thailand Research Fund and the Commission on Higher Education, Thailand. We also want to thank the Hitachi Scholarship Foundation for their support.

(Manuscript received Nov. 24, 2006, revised May 2, 2007)

\section{References}

(1) K. Sakai, S. Tsuru, D. L. Abella, and M. Hara : "Conducting particle motion and particle-initiated breakdown in dc electric field between diverging conducting plates in atmospheric air", IEEE Trans. DEI., Vol.6, pp.122-130 (1999)

(2) T. C. Jordan and M.T. Shaw : "Electrorheology", IEEE Trans. DEI., Vol.24, pp.849-878 (1989)

(3) D. J. Klingenberg, F. van Swoi, and C. F. Zukoski : "Dynamic simulation of electrorheological suspensions", J. Chem. Phys., Vol.91, pp.7888-7895 (1989)

(4) Y. Enomoto and K. Oba : "Simulation of structures and their rheological properties in electrorheological fluids", Physica A., Vol.309, pp.15-25 (2002)

(5) R. Tao and Q. Jiang : "Simulation of structure formation in an electrorheological fluid”, Phys. Rev. Lett., Vol.73, pp.205-208 (1994)

(6) D. J. Klingenberg, F. van Swoi, and C. F. Zukoski : "The small shear rate response of electrorheological suspensions. II. Extension beyond the point-dipole limit”, J. Chem. Phys., Vol.94, pp.6170-6178 (1991)

(7) H. J. H. Clercx and G. Bossis : "Many-body electrostatic interactions in electrorheological fluids", Phys. Rev. E., Vol.48, pp.2721-2738 (1993)

(8) M. Washizu and T. B. Jones : "Multipolar dielectrophoretic force calculation", J. Electrostat., Vol.33, pp.187-198 (1994)

(9) B. Techaumnat, B. Eua-arporn, and T. Takuma : "Calculation of electric field and dielectrophoretic force on spherical particle in chain", J. Appl. Phys., Vol.95, pp.1586-1593 (2004)

(10) M. Washizu and T. B. Jones : "Dielectrophoretic Interaction of Two Spherical Particles Calculated by Equivalent Multipole-Moment Method", IEEE Trans. Ind. Applicat., Vol.32, pp.233-242 (1996)

(11) B. Techaumnat, B. Eua-arporn, and T. Takuma : "Electric field and dielectrophoretic force on a dielectric particle chain in a parallel-plate electrode system", J. Phys. D: Appl. Phys., Vol.37, pp.3337-3346 (2004)

(12) A. Limsimarat and B. Techaumnat : "Electric field and force on a dielectric particle between two diverging plate electrodes", J. Electrostat., Vol.63, pp.789-794 (2005) 


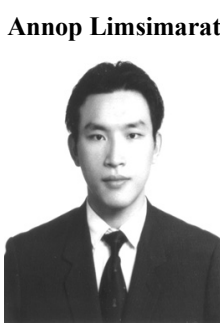

electromagnetics.
(Non-member) was born in Bangkok, Thailand, on September 9, 1974. He graduated with B.Eng. and M.Eng. degrees in electrical engineering from Chulalongkorn University, Thailand, in 1996 and 1999, respectively. Currently, he is working for Ph.D. degree at Chulalongkorn University. His main research fields are high voltage engineering, dynamic simulation of electrorheological fluids and numerical
Boonchai Techaumnat (Member) was born in Bangkok, Thailand, on July 24,1970 . He received the B.Eng. and M.Eng. degrees in electrical engineering from Chulalongkorn University, Thailand, in 1990 and 1995, respectively. He received the D.Eng. in electrical engineering from Kyoto University, Japan, in 2001. He joined the Faculty of Engineering, Chulalongkorn University as a lecturer in 1995, and has been an Assistant Professor at the faculty since 2003. Dr. Techaumnat received the medal prize for new scholars from the Thailand Research Fund in 2005. His research interests include numerical field analysis, electrical insulation, and bioelectromagnetics. 